\title{
THE CHROMATIC NUMBER OF KNESER HYPERGRAPHS
}

\author{
N. ALON, P. FRANKL, AND L. LOVÁSZ
}

\begin{abstract}
Suppose the $r$-subsets of an $n$-element set are colored by $t$ colors.
THEOREM 1.1. If $n \geq(t-1)(k-1)+k \cdot r$, then there are $k$ pairwise disjoint $r$-sets having the same color. This was conjectured by Erdös [E] in 1973.

Let $T(n, r, s)$ denote the Turán number for s-uniform hypergraphs (see $\S 1$ ).

THEOREM 1.3. If $\varepsilon>0, t \leq(1-\varepsilon) T(n, r, s) /(k-1)$, and $n>n_{0}(\varepsilon, r, s, k)$, then there are $k r$-sets $A_{1}, \bar{A}_{2}, \ldots, A_{k}$ having the same color such that $\left|A_{i} \cap A_{j}\right|<s$ for all $1 \leq i<j \leq k$. If $s=2, \varepsilon$ can be omitted.

Theorem 1.1 is best possible. Its proof generalizes Lovász' topological proof of the Kneser conjecture (which is the case $k=2$ ). The proof uses a generalization, due to Bárány, Shlosman, and Szücs of the Borsuk-Ulam theorem.

Theorem 1.3 is best possible up to the $\varepsilon$-term (for large $n$ ). Its proof is purely combinatorial, and employs results on kernels of sunflowers.
\end{abstract}

1. Introduction. Let $n, k, r, t, s$ be positive integers and let $X$ be an $n$-element set. We denote by $\left(\begin{array}{l}X \\ r\end{array}\right)$ the collection of all $r$-element subsets of $X$.

Suppose that $n \leq(k r-1)+(t-1)(k-1)$ and write $X=X_{0} \cup \cdots \cup X_{t-1}$, where $\left|X_{0}\right|=k r-1,\left|X_{1}\right|=\cdots=\left|X_{t-1}\right|=k-1$. Define

$$
\mathcal{F}_{0}=\left(\begin{array}{c}
X_{0} \\
r
\end{array}\right), \quad \mathcal{F}_{i}=\left\{F \in\left(\begin{array}{c}
X \\
r
\end{array}\right): F \cap X_{i} \neq \varnothing\right\}, \quad i=1, \ldots, t-1 .
$$

It is easy to check that none of these families contains $k$ pairwise disjoint members, moreover, $\mathcal{F}_{0} \cup \cdots \cup \mathcal{F}_{t-1}=\left(\begin{array}{l}X \\ r\end{array}\right)$.

Our first result states that such a partition does not exist for $n>(k r-1)+$ $(t-1)(k-1)$.

THEOREM 1.1. Suppose that $n \geq k r+(t-1)(k-1)$ and $\left(\begin{array}{l}X \\ r\end{array}\right)$ is partitioned into $t$ families. Then one of the families contains $k$ pairwise disjoint $r$-element sets.

For $k=2$ the statement of the theorem was conjectured by Kneser $[\mathbf{K n}]$ and proved by Lovász [L1] (cf. also [Bá]). The validity of Theorem 1.1 was conjectured by Erdös $[\mathbf{E}]$ in 1973 (cf. also [Gy]). The case $r=2$ was proved by Cockayne and Lorimer $[\mathbf{C L}]$ and independently by Gyárfás $[\mathbf{G y}]$. The case $t=2$ was proved in $[\mathbf{A F}]$.

Theorem 1.1 immediately implies the following extension.

COROLlaRY 1.2. Suppose $k_{1} \geq \cdots \geq k_{t} \geq 2$ and $n \geq k_{1} r+\sum_{2 \leq i \leq t}\left(k_{i}-1\right)$. If $\left(\begin{array}{l}X \\ r\end{array}\right)=\mathcal{F}_{1} \cup \cdots \cup \mathcal{F}_{t}$, then for some $i, 1 \leq i \leq t$, the family $\mathcal{F}_{i}$ contains $k_{i}$ pairwise disjoint members.

Received by the editors October 18, 1985.

1980 Mathematics Subject Classification (1985 Revision). Primary 05A05; Secondary 05B40, $05 \mathrm{C} 65,55 \mathrm{~A} 15$. 
ProOF. Let $Y_{2}, \ldots, Y_{t}$ be pairwise disjoint sets which are disjoint from $X$, with cardinality $\left|Y_{i}\right|=k_{1}-k_{i}$. Set $X^{\prime}=X \cup Y_{2} \cup \cdots \cup Y_{t} ; \bar{F}_{1}^{\prime}=\mathcal{F}_{1}$ and $\mathcal{F}_{i}^{\prime}=\mathcal{F}_{i} \cup\{F \in$ $\left.\left(\begin{array}{c}X^{\prime} \\ r\end{array}\right): F \cap Y_{i} \neq \varnothing\right\}, i=2, \ldots, t$. Now an application of Theorem 1.1 to $\left(\begin{array}{c}X^{\prime} \\ r\end{array}\right)$ gives the result.

Bárány suggested the following problem. What is the maximal number $m$ so that whenever $\left(\begin{array}{c}X \\ r\end{array}\right)$ is partitioned into $m$ classes one of the classes contains $k r$-sets with all pairwise intersections of size less than $s$. The case $s=1$ is settled by Theorem 1.1.

Suppose $r>s \geq 2$. Let $S \subset\left(\begin{array}{c}X \\ s\end{array}\right)$ be such that for every $H \in\left(\begin{array}{c}X \\ r\end{array}\right)$ there exists $S \in S$ with $S \subset H$, i.e., $S$ contains no independent set of size $r$. The minimum possible size of such $S$ is denoted by $T(n, r, s)$. The problem of determining $T(n, r, s)$ was raised by Turán $[\mathbf{T 1}, \mathbf{T 2}]$ who settled the case $s=2$. In that case the only extremal graph is the disjoint union of $r-1$ complete graphs of nearly equal sizes. In the general case the exact value of $T(n, r, s)$ is unknown. Katona, Nemetz, and Simonovits [KNS] proved that $T(n, r, s) /\left(\begin{array}{c}n \\ s\end{array}\right)$ is monotone increasing as a function of $n$ and thus $t(r, s)=\lim _{n \rightarrow \infty} T(n, r, s) /\left(\begin{array}{c}n \\ s\end{array}\right)$ exists. However, the value of $t(r, s)$ is unknown for all $r>s \geq 3$.

Let $S \subset\left(\begin{array}{c}X \\ s\end{array}\right),|S|=T(n, r, s)$, and assume that each $H \in\left(\begin{array}{l}X \\ r\end{array}\right)$ contains a member of $S$. Partition $S$ into $m=\lceil T(n, r, s) /(k-1)\rceil$ subfamilies $S_{1}, \ldots, S_{m}$ with $\left|S_{i}\right| \leq k-1$. Define $\mathcal{F}_{i}$ to be the collection of those $r$-subsets of $X$ which contain some member of $S_{i}$, i.e.,

$$
\mathcal{F}_{i}=\left\{F \in\left(\begin{array}{c}
X \\
r
\end{array}\right): \exists S \in S_{i}, S \subset F\right\}, \quad i=1, \ldots, m .
$$

One readily checks that $\mathcal{F}_{1} \cup \cdots \cup \mathcal{F}_{m}=\left(\begin{array}{c}X \\ r\end{array}\right)$ and none of the $\mathcal{F}_{i}$ contains $k$ sets whose pairwise intersections have less than $s$ elements.

Our next result shows that this is essentially best possible.

THEOREM 1.3. Suppose $\left(\begin{array}{c}X \\ r\end{array}\right)=\mathcal{F}_{1} \cup \cdots \cup \mathcal{F}_{m}$ and for all $i, 1 \leq i \leq m$, and for arbitrary $k$ sets $F_{1}, \ldots, F_{k} \in \mathcal{F}_{i}$ there exist $1 \leq a<b \leq k$ so that $\left|\bar{F}_{a} \cap F_{b}\right| \geq s$. Then for $r, s, k$ fixed and $n \rightarrow \infty$.

(1) $m \geq(1-o(1)) T(n, r, s) /(k-1)$.

Also if $s=2$ and $n>n_{0}(k, r)$, then

(2) $m \geq T(n, r, 2) /(k-1)$.

The case $k=2$ of the above theorem was proved by Frankl [F2] (cf. also [FF]).

The proof of Theorem 1.1 is topological and uses some of the ideas of $[\mathbf{L 1}]$, whereas the proof of Theorem 1.3 is purely combinatorial.

The paper is organized as follows. In $\S 2$ an outline of the proof of Theorem 1.1 is given. The actual arguments are contained in $\S \S 3$ and 4 . The proof of Theorem 1.3 is given in $\S \S 5$ and 6 . $\S 7$ contains some final remarks.

2. An outlined proof of Theorem 1.1. The basic ideas in the proof of Theorem 1.1 are similar to those used by Lovász in $[\mathbf{L 1}]$, but there are several additional complications. We outline the arguments below and then discuss each step in full in the following two sections.

First it is useful to reformulate Theorem 1.1 in terms of the chromatic number of the Kneser hypergraph. 
Let $G_{n, k, r, s}$ be the $k$-uniform Kneser hypergraph defined as follows. The vertices of $G$ are all the $r$-subsets of $\{1,2, \ldots, n\}$, and a collection of $k$ vertices forms an edge if each pair of the corresponding $r$-sets have intersection of cardinality smaller than s. Put also $G_{n, k, r, 1}=G_{n, k, r}$. Theorem 1.1 is equivalent to the statement that if $n \geq(t-1)(k-1)+k r$, then $G_{n, k, r}$ is not $t$-colorable.

For any $k$-uniform hypergraph $H=(V, E)$, define a simplicial complex $C(H)$ as follows: the vertices of $C(H)$ are all the $|E| k$ ! ordered $k$-tuples $\left(v_{1}, v_{2}, \ldots, v_{k}\right)$ of vertices of $H$, where $\left\{v_{1}, \ldots, v_{k}\right\} \in E$. A set of vertices $\left(v_{1}^{i}, \ldots, v_{k}^{i}\right)_{i \in I}$ of $C(H)$ forms a simplex if there is a complete $k$-partite subgraph of $H$ on the (pairwise disjoint) sets of vertices $V_{1}, V_{2}, \ldots, V_{k}$ such that $v_{j}^{i} \in V_{j}$ for all $i \in I$ and $1 \leq j \leq k$.

Recall that for $s \geq 0$, a topological space $T$ is s-connected if for all $0 \leq l \leq s$, every continuous mapping of the $l$-dimensional sphere $S^{l}$ into $T$ can be extended to a continuous mapping of the $l+1$-dimensional ball $B^{l+1}$ with boundary $S^{l}$ into $T$. Thus 0-connected means arcwise connected, and 1-connected means arcwise connected and simply connected. It will be convenient to agree that $(-1)$-connected means nonempty, and that every space is $s$-connected for all $s<-1$. Theorem 1.1 now follows from the following three assertions.

Proposition 2.1. For any $k$-uniform hypergraph $H$, where $k$ is an odd prime, if $C(H)$ is $((t-1)(k-1)-1)$-connected, then $H$ is not $t$-colorable.

Proposition 2.2. $C\left(G_{n, k, r}\right)$ is $(n-k r-1)$-connected. Thus if $n \geq(t-1)$. $(k-1)+k r$, then it is $((t-1)(k-1)-1)$-connected.

Proposition 2.3. The validity of Theorem 1.1 for $(r, t, k)$ and $\left(r^{\prime}=(t-1)\right.$. $\left.(k-1)+k r, t, k^{\prime}\right)$ implies its validity for $\left(r, t, k k^{\prime}\right)$.

Proposition 2.1 appears interesting in its own right. It probably holds for every positive integer $k$. If we replace Lemma 3.1 below by the Borsuk-Ulam theorem then the proof given in $\S 3$ shows its validity for $k=2$. (This, in fact, easily follows from Lovász' regult [L1].) At the moment we cannot prove Proposition 2.1 for nonprime $k$.

Propositions 2.1 and 2.2 imply the assertion of Theorem 1.1 for every odd prime $k$. By Lovász's proof of the Kneser conjecture [L1], Theorem 1.1 holds for $k=2$. Thus, by Proposition 2.3, Theorem 1.1 holds for all $r, t, k$.

Proposition 2.1 is derived in $\S 3$ from an extension, due to Bárány, Shlosman, and Szücs [BSS], of the well-known Borsuk-Ulam theorem of algebraic topology.

Proposition 2.2 is proved in $\S 4$ using several standard results from topology.

We conclude this section with the (easy) proof of Proposition 2.3.

ProOF of Proposition 2.3. Suppose $n \geq(t-1)\left(k k^{\prime}-1\right)+r k k^{\prime}$ and let $c$ be a coloring of the $r$-subsets of $N=\{1,2, \ldots, n\}$ by $t$ colors. Put $r^{\prime}=(t-1)(k-1)+r k$ and define a coloring $c^{\prime}$ of the $r^{\prime}$-subsets of $N$ by $t$ colors as follows. Let $A$ be an $r^{\prime}$-subset of $N$. By Theorem 1.1 (for $\left.r, t, k\right) A$ contains $k$ pairwise disjoint $r$-sets of the same color. Color $A$ by the first such color. Notice that $n \geq(t-1)\left(k^{\prime}-1\right)+k^{\prime} \cdot r^{\prime}$ and hence by Theorem 1.1 (for $r^{\prime}, t, k^{\prime}$ ) there are $k^{\prime}$ pairwise disjoint $r^{\prime}$-subsets of $N$ having the same color. Each of these subsets contains $k$ pairwise disjoint $r$-subsets used in defining its color, and all these $k^{\prime} \cdot k$ pairwise disjoint $r$-sets have the same color. 
3. The chromatic number of $H$ and the connectivity of $C(H)$. We begin by stating a result of Bárány, Shlosman, and Szücs from [BSS]. Let $k$ be an odd prime, and suppose $m \geq 1$. Let $X=X_{m, k}$ denote the CW-complex consisting of $k$ disjoint copies of the $m(k-1)$-dimensional ball with an identified boundary $S^{m(k-1)-1}$. We define a free action of the cyclic group $Z_{k}$ on $X$ by defining $\omega$, the action of its generator, as follows (see [Bou, 13]). Represent $S^{m(k-1)-1}$ as the set of all $m$ by $k$ real matrices $\left(a_{i j}\right)$ satisfying

$$
\sum_{j=1}^{k} a_{i j}=0 \quad \text { for all } 1 \leq i \leq m \quad \text { and } \quad \sum_{i, j} a_{i j}^{2}=1 .
$$

Define now $\omega\left(a_{i j}\right)=\left(a_{i, j+1}\right)$, where $j+1$ is reduced modulo $k$. Thus $\omega$ just cyclically shifts the columns of a matrix representing a point of $S^{m(k-1)-1}$. Trivially, this action is free, i.e., $\omega(x) \neq x$ for all $x \in S^{m(k-1)-1}$. The map $\omega$ is extended from $S^{m(k-1)-1}$ to $X_{m, k}$ as follows. Let $(y, r, q)$ denote a point of $X_{m, k}$ from the $q$ th ball with radius $r$ and $S^{m(k-1)-1}$-coordinate $y$. Then

$$
\omega(y, r, q)=(\omega y, r, q+1),
$$

where $q+1$ is reduced modulo $k$. One can easily check that $\omega$ defines a free $Z_{k}$ action on $X=X_{m, k}$.

The following result of Bárány, Shlosman, and Szücs is crucial for the proof of Proposition 2.1.

LEMMA 3.1 [BSS]. For any continuous map $h: X \rightarrow R^{m}$ there exists an $x \in X$ such that $h(x)=h(\omega x)=\cdots=h\left(\omega^{k-1} x\right)$.

Let $H$ be a $k$-uniform hypergraph and let $C(H)$ be the associated simplicial complex defined in $\S 2$. Define a free $Z_{k}$ action on $C(H)$ by defining $\gamma$, the action of its generator, as follows. For a vertex $\left(v_{1}, v_{2}, \ldots, v_{k}\right)$ of $C(H), \gamma\left(v_{1}, v_{2}, \ldots, v_{k}\right)=$ $\left(v_{2}, \ldots, v_{k}, v_{1}\right)$. Over the other points of $C(H), \gamma$ is extended by linearity.

Let $X$ and $Y$ be topological spaces and assume that $Z_{k}$ acts freely on both. Let $\alpha$ and $\beta$ denote the action of the generator of $Z_{k}$ on $X$ and $Y$, respectively. We say that a continuous mapping $f: X \rightarrow Y$ is $Z_{k}$-equivariant if $f \circ \alpha=\beta \circ f$ (cf. [Bou, Chapter 13]).

LEMMA 3.2. If $C(H)$ is $(m(k-1)-1)$-connected, then there exists a $Z_{k}$ equivariant map $f: X_{m, k} \rightarrow C(H)$.

ProOF. Given an equivariant cell subdivision of $X=X_{m, k}$ we construct $f$ by induction on the dimension of the cells. (We note that we can, using certain circulant matrices, explicitly define such a cell subdivision (containing precisely $k$ cells, i.e., one orbit, of each dimension $i, 0 \leq i \leq m(k-1)=\operatorname{dim} X)$, but this is not required here.)

We first choose a 0 -cell (= vertex) from each orbit of 0 -cells, define $f$ on these vertices arbitrarily, and extend the map to a $Z_{k}$-equivariant map of all vertices. Assuming $f$ has already been defined on the $(i-1)$-skeleton of $X(0<i \leq m(k-1))$, define it on the $i$-cells as follows. Choose a cell from each orbit of the $i$-cells. (Note that from the freeness of the action it follows that each orbit of $i$-cells contains $k$ members.) Now $f$ is defined on the boundary of these cells and thus, since $X$ is $(i-1)$-connected it can be extended continuously to those chosen $i$-cells. Now 
extend $f$ to a $Z_{k}$-equivariant map of all the $i$-cells. This completes the proof of the lemma.

Let $Y=R^{(t-1)(k-1)}-\{\bar{o}\}$ be represented by the set of all $t$ by $k$ real matrices $\left(a_{i j}\right)$ satisfying

$$
\begin{aligned}
& \sum_{j=1}^{k} a_{i j}=0 \quad \text { for all } 1 \leq i \leq t, \\
& \sum_{i=1}^{t} a_{i j}=0 \quad \text { for all } 1 \leq j \leq k \text { and } \quad \sum_{i, j} a_{i j}^{2}>0 .
\end{aligned}
$$

Clearly, there is a free $Z_{k}$ action on $Y$; its generator, $\beta$, maps $\left(a_{i j}\right) \in Y$ to $\left(a_{i, j+1}\right) \in Y$, where $j+1$ is reduced modulo $k$.

LEMMA 3.3. If $H$ is $t$-colorable, then there exists a $Z_{k}$-equivariant map $g: C(H)$ $\rightarrow Y$.

PROOF. For a real vector $u$ of length $k$ and for $1 \leq i \leq t$, let $R_{i}(u)$ denote the $t$ by $k$ matrix whose $i$ th row is $u$ and all other rows are the zero vector. If $u=\left(u_{1}, u_{2}, \ldots, u_{k}\right)$ define $\sigma u=\left(u_{k}, u_{1}, \ldots, u_{k-1}\right)$. Let $z$ be the following vector of length $k ; z=(1,-1,0, \ldots, 0)$. Notice that $\sum_{i=0}^{k-1} \sigma^{i} z=0$ and this is the only nontrivial linear relation between the vectors $\sigma^{i} z$.

Let $H=(V, E)$ be a $t$-colorable hypergraph and let $c: V \rightarrow\{1,2, \ldots, t\}$ be a proper $t$-coloring of it. We define $g: C(H) \rightarrow Y$ by defining it on the vertices of $C(H)$ and extending it by linearity. For a vertex $\left(v_{1}, v_{2}, \ldots, v_{k}\right)$ of $C(H)$, put

$$
g\left(v_{1}, v_{2}, \ldots, v_{k}\right)=\sum_{i=1}^{k} R_{c\left(v_{i}\right)}\left(\sigma^{i} z\right)
$$

Notice that since $\left\{v_{1}, \ldots, v_{k}\right\}$ is an edge of $H$ and $c$ is a proper coloring, not all the $c\left(v_{i}\right)$ 's are the same and thus the right-hand side of (3.1) is a nonzero matrix. One can easily check that this matrix has zero row and column sums and thus belongs to $Y$. It is not difficult to check that $g$ is equivariant, i.e., $g \circ \gamma(x)=\beta \circ g(x)$ for all $x \in C(H)$. This easily holds for all vertices $x$ of $C(H)$ and hence for all $x \in C(H)$. It remains to show that $g(x) \in Y$ for all $x \in C(H)$. Clearly $g(x)$ is a $t$ by $k$ matrix with zero row and column sums. We must check that it is a nonzero matrix. The point $x$ is a convex combination $\sum_{i \in I} \lambda_{i}\left(v_{1}^{i}, \ldots, v_{k}^{i}\right)$ of the vertices of some face of $C(H), \sum \lambda_{i}=1, \lambda_{i}>0$ for all $i \in I$. By definition there are $k$ pairwise disjoint subsets $V_{1}, \ldots, V_{k}$ of vertices of $H$ such that $v_{j}^{i} \in V_{j}$ for all $1 \leq j \leq k$ and $i \in I$, and all the $\prod_{j=1}^{k}\left|V_{j}\right|$ edges $\left(w_{1}, \ldots, w_{k}\right)$, where $w_{j} \in V_{j}$ are edges of $H$. Since $c$ is a proper coloring of $H$, this means that every color is missing from at least one of the $V_{j}$ 's. By definition

$$
g(x)=\sum_{i \in I} \lambda_{i} \sum_{j=1}^{k} R_{c\left(v_{j}^{i}\right)}\left(\sigma^{j} z\right) .
$$

We claim that the $c\left(v_{j}^{i}\right)$ th row of this matrix is nonzero for each $i \in I$ and $1 \leq j \leq k$. Indeed, this row is a combination, with positive coefficients, of the vectors $\sigma^{j} z$ for 
all $j$ 's such that $c\left(v_{j}^{i}\right)$ appears as a color of some vertex in $V_{j}$. These are not all the $\sigma^{j} z$ 's and hence such a combination cannot be zero. This completes the proof.

To prove Proposition 2.1 we need one more observation.

LEMMA 3.4. Let $Y=R^{(t-1)(k-1)}-\{\bar{o}\}$ and let $\beta$ be as above. Then there is a continuous map $h: Y \rightarrow R^{t-1}$ such that no $y \in Y$ satisfies $h(y)=h(\beta y)=\cdots=$ $h\left(\beta^{k-1} y\right)$.

ProOF. For $y=\left(a_{i j}\right)_{1 \leq i \leq t, 1 \leq j \leq k}, y \in Y$, define $h(y)=\left(a_{i 1}\right)_{1 \leq i \leq t}$. Clearly $h$ maps $Y$ into a $t-1$ dimensional space (since $\sum_{i=1}^{t} a_{i 1}=0$ for each $y=\left(a_{i j}\right) \in Y$ ). If $y=\left(a_{i j}\right) \in Y$ and $h(y)=h(\beta y)=\cdots=h\left(\beta^{k-1} y\right)$, then $a_{i 1}=a_{i 2}=\cdots=a_{i k}$ for all $1 \leq i \leq t$, and since $\sum_{j=1}^{k} a_{i j}=0$ we conclude that $a_{i j}=0$ for all $i, j$, contradicting the definition of $Y$. This completes the proof.

ProOF OF PROPOSITION 2.1. Let $k$ be an odd prime and let $H$ be a $k$ uniform hypergraph whose associated simplicial complex $C(H)$ is $((t-1)(k-1)-1)$ connected. We must show that $H$ is not $t$-colorable. Suppose $H$ is $t$-colorable. Since $C(H)$ is $((t-1)(k-1)-1)$-connected, Lemma 3.2 implies that there exists an equivariant $f: X_{t-1, k} \rightarrow C(H)$. By Lemma 3.3 and the assumption that $H$ is $t$-colorable, there exists an equivariant $g: C(H) \rightarrow Y=R^{(t-1)(k-1)}-\{\bar{o}\}$. Finally, let $h: Y \rightarrow R^{t-1}$ be as in Lemma 3.4 and put $F=h \circ g \circ f: X_{t-1, k} \rightarrow R^{t-1}$. $F$ is clearly continuous. We claim that there is no $x \in X=X_{t-1, k}$ such that $F(x)=F(\omega x)=\cdots=F\left(\omega^{k-1} x\right)$. Indeed, if $x \in X$ satisfies the above, then by the equivariance of $f$ and $g, y=g \circ f(x)$ would satisfy $h(y)=h(\beta y)=\cdots=h\left(\beta^{k-1} y\right)$, contradicting Lemma 3.4. Thus the claim holds, and this contradicts Lemma 3.1. Therefore our assumption was false and $H$ is not $t$-colorable, as claimed.

4. The connectivity of $C\left(G_{n, k, r}\right)$. Let $G=G_{n, k, r}$ be the $k$-uniform Kneser hypergraph defined in $\S 2$, and let $C(G)$ be the corresponding simplicial complex. The vertices of $C(G)$ are ordered $k$-tuples $\left(R_{1}, \ldots, R_{k}\right)$ of pairwise disjoint $r$-sets of $N=\{1,2, \ldots, n\}$, and a set of such $k$-tuples $\left(R_{1}^{i}, R_{2}^{i}, \ldots, R_{k}^{i}\right)_{i \in I}$ forms a simplex if there exists an ordered partition of $N$ into $k$ pairwise disjoint parts $N=N_{1} \cup$ $\cdots \cup N_{k}$, and $R_{j}^{i} \subseteq N_{j}$ for all $i \in I$ and $1 \leq j \leq k$. In this section we show that $C(G)$ is $(n-k r-1)$-connected. We need a few known results from topology. For a (finite) family of sets $\mathcal{F}$, the nerve of $\mathcal{F}$ is a simplicial complex whose vertices are the members of $F$ of $\mathcal{F}$ and a set $\left(F_{i}\right)_{i \in I}$ of members of $\mathcal{F}$ forms a face if $\bigcap_{i \in I} F_{i} \neq \varnothing$.

The following is a classical result (cf. [Bo, BKL]).

LEMMA 4.1 (NERVE THEOREM). Let $C$ be a simplicial complex and $N$ the nerve of the family of its maximal faces. Then $N$ and $C$ are homotopy equivalent (and thus have the same connectivity).

LEMMA 4.2. Let $C_{1}$ and $C_{2}$ be two simplicial complexes. If $C_{1}, C_{2}$ are both $s$ connected, and their intersection $C_{1} \cap C_{2}$ is $(s-1)$-connected, then the union $C_{1} \cup C_{2}$ is s-connected. (Recall that by definition (-1)-connected means nonempty.)

PROOF. This well-known result follows from the Mayer-Vietoris long exact sequence together with the Van Kampen and Hurewicz theorems. It also follows from Lemmas 4.8 and 4.9 in [BKL]. 
COROLlaRY 4.3. Let $C_{1}, C_{2}, \ldots, C_{m}$ be simplicial complexes. If the intersection of any family of $l \geq 1$ of them is $(s-l+1)$-connected, then $C_{1} \cup C_{2} \cup \cdots \cup C_{m}$ is s-connected.

Proof. We use induction on $m$. For $m=2$ this is Lemma 4.2. Assuming the assertion holds for $m-1$ (and every $s$ ) we prove it for $m(m>2)$. By the induction hypothesis $C \equiv C_{1} \cup \cdots \cup C_{m-1}$ is $s$-connected, and so is $C_{m}$. Now $C \cap C_{m}=\left(C_{1} \cap C_{m}\right) \cup \cdots \cup\left(C_{m-1} \cap C_{m}\right)$ is a union of $m-1(s-1)$-connected simplicial complexes, the intersection of any $l$ of which is $s-l=((s-1)-l+1)$ connected. Thus, by the induction hypothesis, $C \cap C_{m}$ is $(s-1)$-connected and by Lemma $4.2 C \cup C_{m}=C_{1} \cup \cdots \cup C_{m}$ is $s$-connected. This completes the induction and the proof.

For nonnegative integers $n, r_{1}, r_{2}, \ldots, r_{k}$, let $C=C\left(n, r_{1}, \ldots, r_{k}\right)$ denote the following simplicial complex. The vertices of $C$ are all the ordered partitions $\left(N_{1}, \ldots, N_{k}\right)$ of $N=\{1, \ldots, n\}$ into $k$ parts such that $\left|N_{j}\right| \geq r_{j}$ for $1 \leq j \leq k$. A family $\left(N_{1}^{i}, \ldots, N_{k}^{i}\right)_{i \in I}$ of such partitions forms a face if $\left|\bigcap_{i \in I} N_{j}^{i}\right| \geq r_{j}$ for each $1 \leq j \leq k$.

LEMMA 4.4. $C=C\left(n, r_{1}, \ldots, r_{k}\right)$ is $\left(n-\sum_{j=1}^{k} r_{j}-1\right)$-connected.

PROOF. The lemma clearly holds for $n \leq \sum_{j=1}^{k} r_{j}$. For the general case we prove it by induction on $n$. For $n=1$ the result is trivial. Assuming it holds for all $n^{\prime}<n$ we prove it for $n$. If $r_{1}=r_{2}=\cdots=r_{k}=0$, then every set of vertices of $C=C\left(n, r_{1}, \ldots, r_{k}\right)$ forms a face and $C$ is $l$-connected for every $l$. Thus we can assume, without loss of generality, that $r_{1}>0$. For $1 \leq i \leq n$, let $C_{i}$ denote the induced subcomplex of $C$ on the set of all vertices $\left(N_{1}, \ldots, N_{k}\right)$ of $C$ with $i \in N_{1}$. Clearly $C=C_{1} \cup C_{2} \cdots \cup C_{n}$. Put $s=n-\sum_{j=1}^{k} r_{j}-1$. Consider the intersection of $l C_{i}-s$. If $l \leq r_{1}$, it is isomorphic to $C\left(n-l, r_{1}-l, r_{2}, \ldots, r_{n}\right)$ and is, by the induction hypothesis, $s$-connected and hence certainly $(s-l+1)$-connected. If $l>r_{1}$ this intersection is isomorphic to $C\left(n-l, 0, r_{2}, \ldots, r_{n}\right)$ and is, by the induction hypothesis, $n-l-\sum_{j=2}^{k} r_{j}-1=s-l+r_{1} \geq(s-l+1)$-connected. Therefore, by Corollary $4.3, C$ is $s$-connected. This completes the induction and the proof.

ProOF OF Proposition 2.2. One can easily check that the nerve of maximal faces of $C\left(G_{n, k, r}\right)$ is $C\left(n, r_{1}, r_{2}, \ldots, r_{k}\right)$, where $r_{j}=r$ for $1 \leq j \leq k$. The proposition thus follows from Lemma 4.1 and Lemma 4.4.

5. Families of $r$-sets without $k$ members with mutually small intersection. To avoid long sentences like the title of this section, let us say that $\mathcal{F}$ has property $P(k, s)$ or shortly $\mathcal{F}$ has $P(k, s)$ if there are no sets $F_{1}, F_{2}, \ldots, F_{k} \in \mathcal{F}$ satisfying $\left|F_{i} \cap F_{j}\right|<s$ for $1 \leq i<j \leq k$.

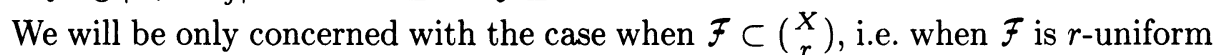
and $|X|=n>n_{0}(k, r, s)$. Then $P(k, s)$ makes sense only for $1 \leq s<r$, which we suppose. Also, we assume that $k \geq 2$.

The simplest way of constructing $₹$ having $P(k, s)$ is the following. Let $A_{1}, \ldots$, $A_{l}$ be distinct $s$-element subsets of $X$. Define $\mathcal{F}\left(A_{1}, \ldots, A_{l}\right)=\left\{F \in\left(\begin{array}{c}X \\ r\end{array}\right): \exists i\right.$, $1 \leq i \leq l, A \subset F\}$. It is easy to check that $\mathcal{F}\left(A_{1}, \ldots, A_{l}\right)$ has $P(k, s)$ whenever 
$l \leq k-1$ and

$(k-1)\left(\begin{array}{c}n-s \\ r-s\end{array}\right)-\left(\begin{array}{c}k-1 \\ 2\end{array}\right)\left(\begin{array}{l}n-s-1 \\ r-s-1\end{array}\right) \leq\left|\mathcal{F}\left(A_{1}, \ldots, A_{k-1}\right)\right| \leq(k-1)\left(\begin{array}{c}n-s \\ r-s\end{array}\right)$.

It is not hard to see that $\left|\mathcal{F}\left(A_{1}, \ldots, A_{k-1}\right)\right|$ is maximal if $A_{1}, \ldots, A_{k-1}$ are pairwise disjoint. Hajnal and Rothschild $[\mathbf{H R}]$ proved that this provides the maximum size of any $\mathcal{F} \subset\left(\begin{array}{c}X \\ r\end{array}\right)$ having $P(k, s)$ for $n>n_{0}(k, r, s)$.

Let us mention that the special case $k=2$ is the Erdös-Ko-Rado theorem [EKR].

We need a strengthening of the Hajnal-Rothschild theorem. A similar strengthening of the Erdös-Ko-Rado theorem was given in $[\mathbf{F 1}]$.

THEOREM 5.1. Suppose $\mathcal{F} \subset\left(\begin{array}{l}X \\ r\end{array}\right)$ and $\mathcal{F}$ has property $P(k, s)$. Then there exists an $l, 0 \leq l<k$, and a family $\mathcal{A}=\left\{A_{1}, \ldots, A_{l}\right\}$ of $s$-element sets so that

$\left|\mathcal{F}-\mathcal{F}\left(A_{1}, \ldots, A_{l}\right)\right|=\left|\left\{F \in \mathcal{F}: \nexists i, A_{i} \subset F\right\}\right| \leq \sum_{i=s+1}^{r}\left(\begin{array}{c}n-i \\ k-i\end{array}\right) i !(k-1)^{i} r^{i(i-s+1)}$.

Moreover, if $l=k-1$, then $\mathcal{F} \subset \mathcal{F}\left(A_{1}, \ldots, A_{k-1}\right)$ holds.

To prove Theorem 5.1 we introduce the family $\xi^{*}$. Let us define $b(j)=$ $(k-1) r+1$ for $j \leq s$ and $b(s+i)=(k-1) r^{i+1}+1$ for $1 \leq i<r-s$. Let $F^{*}$ consist of those subsets $G$ of $X$ for which one can find $b=b(|G|)$ members $F_{1}, \ldots, F_{b}$ of F such that $F_{i} \cap F_{j}=G$ for $1 \leq i<j \leq b$. The collection $\left\{F_{1}, \ldots, F_{b}\right\}$ is called a sunflower with center $G$. The sets $F_{i}-G$ are the petals. Note that they are pairwise disjoint.

Define $B$ as the family of inclusionwise minimal members of $₹ \cup \mathcal{F}^{*}$, i.e.

$$
B=\left\{B \in \mathcal{F} \cup \mathcal{F}^{*}: \nexists B^{\prime} \in \mathcal{F} \cup \mathcal{F}^{*}, B^{\prime} \varsubsetneqq B\right\} .
$$

Note that $B$ is a basis for $\mathcal{F}$, i.e., for every $F \in \mathcal{F}$ there exists $B \in B$ with $B \subseteq F$.

Proposition 5.2. $\mathcal{F} \cup \mathcal{F}^{*}$ (and hence B) has $P(k, s)$.

ProOF. Suppose for contradiction that $F_{1}, \ldots, F_{l}, G_{l+1}, \ldots, G_{k}$ have pairwise intersections of size strictly less than $s, F_{1} \ldots, F_{l} \in \mathcal{F}, G_{l+1}, \ldots, G_{k} \in \mathcal{F}^{*}$, and $l$ is maximal with respect to these assumptions. Since $\mp$ has $P(k, s), l<k$ holds.

By definition $G_{l+1}$ is the center of a sunflower $\left\{\tilde{F}_{1}, \ldots, \tilde{F}_{b}\right\}$ where $b>(k-1) r$, $\tilde{F}_{i} \in \mathcal{F}$. Since $\left|F_{1} \cup \cdots \cup F_{l} \cup G_{l+2} \cup \cdots \cup G_{k}\right| \leq(k-1) r$, this set cannot intersect all $b$ pairwise disjoint petals $\tilde{F}_{i}-G_{l+1}$ of the sunflower. Say $\left(F_{1} \cup \cdots \cup F_{l} \cup G_{l+2} \cup\right.$ $\left.\cdots \cup G_{k}\right) \cap\left(\tilde{F}_{j}-G_{l+1}\right) \neq \varnothing$.

Set $F_{l+1}=\tilde{F}_{j}$ and verify that $F_{1}, \ldots, F_{l+1}, G_{l+2}, \ldots, G_{k}$ have pairwise intersection of size strictly less than $s$, in contradiction with the maximal choice of $l$.

Let $b_{i}$ denote the number of $i$-element members of $B$. The next proposition clearly implies Theorem 5.1.

Proposition 5.3. (i) $b_{i}=0$ for $i<s$,

(ii) $b_{s}<k$,

(iii) $b_{i} \leq i !(k-1)^{i} r^{i(i-s+1)}$ for $s<i \leq r$.

ProOF. To prove (i) note again that if $G \in B$ but $G \notin \mathcal{F}$, then $G$ is the center of a sunflower of size at least $(k-1) r+1 \geq k$, i.e., there exist $F_{1}, \ldots, F_{k} \in \mathcal{F}$ 
satisfying $F_{i} \cap F_{j}=G$ and thus $\left|F_{i} \cap F_{j}\right|=|G|$ for $1 \leq i<j \leq k$. Since $₹$ has $P(k, s),|G| \geq s$, i.e., $b_{i}=0$ for $i<s$.

By Proposition 5.2, $B$ has $P(k, s)$, thus (ii) holds. To prove (iii) we are going to show the $i$-element members of $B$ form no sunflower of size $b(i-1)$.

Suppose for contradiction that $B_{1}, \ldots, B_{b(i-1)}$ are $i$-element sets in $B$ which form a sunflower with center $C$.

We are going to define sets $F_{1}, \ldots, F_{b(i-1)} \in \mathcal{F}$ inductively so that $F_{i} \cap F_{j}=$ $B_{i} \cap B_{j}$ holds for $1 \leq i<j \leq b(i-1)$. Then $\left\{F_{1}, \ldots, F_{b(i-1)}\right\}$ is a sunflower with center $C$ implying $C \in \mathcal{F}^{*}$. However, $C \varsubsetneqq B_{1} \in B$, a contradiction.

So let us suppose that $F_{j^{\prime}}$ was defined for $j^{\prime}<j, j \leq b(i-1), B_{j}$ is the center of a sunflower $\left\{\tilde{F}_{1}, \ldots, \tilde{F}_{b(i)}\right\}$ with $\tilde{F}_{\nu} \in \mathcal{F}$. Consider $A=F_{1} \cup \cdots \cup F_{j-1} \cup B_{j+1} \cup \cdots \cup$ $B_{b(i-1)}$. Then $|A| \leq b(i-1) r<b(i)$. Therefore among the $b(i)$ pairwise disjoint petals $\tilde{F}_{\nu}-B_{j}$ there is one, say $\tilde{F}_{\nu}-B_{j}$, which is disjoint to $A$. Set $F_{j}=\tilde{F}_{\nu}$ and verify that $F_{1}, \ldots, F_{b(i-1)}$ fulfill the requirements.

Now the bound (iii) is a direct consequence of a classical result of Erdös and Rado [ER], which says that any family of more than $i !(b-1)^{i}$ distinct $i$-sets contains a sunflower of size $b$.

Proof of TheOREM 5.1. Set $l=b_{s}$ and let $A=\left\{A_{1}, \ldots, A_{l}\right\}$ be the collection of $s$-element members of $B$. There is a last thing to check, namely that $b_{s}=$ $k-1$ implies $b_{i}=0$ for $i>s$. In fact, if $B \in B,|B|=i>s$, then the sets $A_{1}, \ldots, A_{k-1}$ and $B$ have pairwise intersections of size strictly less than $s\left(A_{i} \not \subset B\right.$ !) in contradiction with Proposition 5.2.

6. The chromatic number of the generalized Kneser hypergraphs. Suppose now $\left(\begin{array}{c}X \\ r\end{array}\right)$ is colored by $t$ colors, i.e., $\left(\begin{array}{c}X \\ r\end{array}\right)=\mathcal{F}_{1} \cup \cdots \cup \mathcal{F}_{t}$, in such a way that none of the $\mathcal{F}_{i}$ 's contains an edge of $G_{n, k, r, s}$. That is $\mathcal{F}_{i}$ has property $P(k, s)$ for $i=1, \ldots, t$.

Apply Theorem 5.1 to $\mathcal{F}_{i}$ to obtain a family $\mathscr{A}^{(i)}$ consisting of at most $k-1 s$ element subsets of $X$ and so that "most" of the members of $\mathcal{F}$ contain at least one of these $s$-sets.

Set $A=A^{(1)} \cup \cdots \cup \mathcal{A}^{(t)}$. Then $|A| \leq(k-1) t$.

Let $\varepsilon$ be an arbitrary positive number. We have to show that for $n>n_{0}(k, r, s, \varepsilon)$ one has $t>(1-\varepsilon) T(n, r, s) /(k-1)$.

Suppose the contrary. Then $|A| \leq(k-1) t \leq(1-\varepsilon) T(n, r, s)$. By the theory of supersaturated graphs (cf. Theorem $1^{*}$ in [ES] or Theorem 3.8 in [FR]) there are at least $\varepsilon_{1} n^{r} r$-element subsets of $X$ which contain no member of $\mathcal{A}$. Let $\mathcal{G}$ be the collection of these sets, i.e.,

$$
\mathcal{G}=\left\{G \in\left(\begin{array}{c}
X \\
r
\end{array}\right): \nexists A \in A, A \subset G\right\} .
$$

However, Theorem 5.1 guarantees that for $n>n_{0}(k, r, s)$

$$
\left|\mathcal{G} \cap \mathcal{F}_{i}\right|<2\left(\begin{array}{c}
n-s-1 \\
r-s-1
\end{array}\right)(s+1) !(k-1)^{s+1} r^{2(s+1)} .
$$

Thus

$$
\varepsilon_{1} n^{r} \leq|\mathcal{G}| \leq \sum_{i=1}^{t}\left|\mathcal{G} \cap \mathcal{F}_{i}\right|<t n^{r-s-1} 2(s+1) !(k-1)^{s+1} r^{2(s+1)} /(r-s-1) ! .
$$


This yields $t>\varepsilon_{2} n^{s+1}$, where $\varepsilon_{2}$ is a positive constant, depending only on $k, r, s$, and $\varepsilon$. Consequently, we obtained $t>T(n, r, s)$ for $n>n_{0}(k, r, s)$, a contradiction, which concludes the proof of (i).

To prove (ii) suppose $s=2$. Let us first recall Turán's theorem. Denote $T(n, r, 2)$ by $T(n, r)$, i.e., $T(n, r)$ is the minimum number of edges in a graph on $n$ vertices and without an independent set of size $r$.

Suppose $n=n_{1}+\cdots+n_{r-1}$,

$$
\left\lfloor\frac{n}{r-1}\right\rfloor \leq n_{i} \leq\left\lceil\frac{n}{r-1}\right\rceil, \quad i=1, \ldots, r-1 .
$$

Let $\tau(n, r)$ be the graph on $n$ vertices which is the vertex disjoint union of $r-1$ complete graphs of respective sizes $n_{1}, \ldots, n_{r-1}$.

TURÁN'S THEOREM [T1]. Suppose $\mathcal{G}$ is a graph on $n$ vertices and with no independent set of $r$ vertices. Then $|\mathcal{G}| \geq|\tau(n, r)|$ with equality holding if and only if $\mathcal{G}$ is isomorphic to $\mathcal{T}(n, r)$.

Turáns theorem clearly implies

$$
T(n, r)=|\tau(n, r)|=\left(\frac{1}{r-1}+o(1)\right)\left(\begin{array}{l}
n \\
2
\end{array}\right) .
$$

Consequently,

$$
T(n, r)-T(n, r+1)=\frac{1+o(1)}{r(r-1)}\left(\begin{array}{l}
n \\
2
\end{array}\right) .
$$

Thus for $n>n_{0}(k, r)$ the first part of Theorem 1.3 implies $|A|>T(n, r+1)$.

We are going to use the following theorem of Bollobás. Let us denote by $m(n, e, r)$ the minimum number of independent sets of size $r$ in a graph on $n$ vertices and $e$ edges.

THEOREM $6.1[\mathbf{B}]$. Suppose $T(n, r)>e \geq T(n, r+1)$. Then

$$
m(n, e, r) \geq \frac{T(n, r)-e}{T(n, r)-T(n, r+1)}\left\lfloor\frac{n}{r}\right\rfloor^{r} .
$$

If $|\mathcal{A}| \geq T(n, r)$, then $t \geq T(n, r) /(k-1)$ follows. We thus assume $|A|<T(n, r)$. Let us renumber the families $\mathcal{F}_{1}, \ldots, \mathcal{F}_{t}$ so that for some number $t_{0}, 0 \leq t_{0} \leq t$, one has $\left|\mathcal{A}^{(i)}\right|=k-1$ if and only if $i \leq t_{0}$. Then $|A|=\sum_{i=1}^{t}\left|\mathcal{A}^{(i)}\right| \leq(k-1) t-\left(t-t_{0}\right)$. Therefore $|A| \leq T(n, r)-\left(t-t_{0}\right)$. Let us define

$$
R=\left\{R \in\left(\begin{array}{c}
X \\
r
\end{array}\right): \nexists A \in A, A \subset R\right\} .
$$

In view of $(6.1)$ one has

$$
|R| \geq\left(t-t_{0}\right)\left\lfloor\frac{n}{r}\right\rfloor r /(T(n, r)-T(n, r+1))>\left(t-t_{0}\right) n^{r-2} r^{-r}
$$

On the other hand $R \subset \bigcup_{i=t_{0}+1}^{t}\left(\mathcal{F}_{i}-\mathcal{F}_{i}\left(\mathcal{A}^{(i)}\right)\right)$. Applying Theorem 5.1 with $s=2$ gives

$$
|R| \leq\left(t-t_{0}\right) 12(k-1)^{3} r^{6}\left(\begin{array}{c}
n \\
r-3
\end{array}\right) \quad \text { for } n>n_{0}(k, r)
$$


which contradicts (6.2) and thus concludes the proof of the theorem.

REMARK. From the proof it is clear for large $n$ that $t=T(n, r) /(k-1)$ can hold only if $t_{0}=t$ and $A$ is the edge set of the corresponding Turán graph, i.e., the disjoint union of $r-1$ complete graphs with almost equal sizes. That is, there is basically a unique coloring.

7. Concluding remarks. (1) Notice that both Theorem 1.1 and Corollary 1.2 are best possible for all possible values of parameters. Theorem 1.3(ii) is best possible only for large $n$ and the $\varepsilon$-term in Theorem 1.3(i) is probably unnecessary. It would be interesting (but appears difficult) to find the exact chromatic number of $G_{n, k, r, s}$ for all possible $n, k, r, s$.

(2) Lovász's proof for the Kneser conjecture supplied some other applications (see [L2]). It seems that our proof of Theorem 1.1, and especially Proposition 2.1, might yield some further consequences besides Theorem 1.1. It turns out that a very similar method can be used to prove the following result conjectured in [AW] (see also $[\mathbf{G W}]$ ).

Let $N$ be an opened necklace consisting of $n a_{i}$ beads of color $i, 1 \leq i \leq k$. Then it is possible to cut $N$ in at most $(n-1) k$ places and to divide the resulting pieces into $n$ classes, such that each class will contain precisely $a_{i}$ beads of color $i$, $1 \leq i \leq k$. This will appear in $[\mathbf{A 1}]$.

(3) As shown in $\S 1$, if $n=(t-1)(k-1)+k r-1$, then there is a coloring of the $r$-subsets of an $n$-element set such that no $k$ pairwise disjoint $r$-sets have the same color. One can easily check that this coloring is not unique, in fact there are many optimal colorings. This is in sharp contrast with Theorem 1.3.

ACKNOWLEDGMENT. The authors are indebted to M. Saks and P. D. Seymour for stimulating discussions.

\section{REFERENCES}

[AF] N. Alon and P. Frankl, Families in which disjoint sets have large union, Ann. New York Acad. Sci. (to appear).

[A1] N. Alon, Splitting necklaces, Adv. in Math. (to appear).

[AW] N. Alon and D. B. West, The Borsuk-Ulam Theorem and bisection of necklaces, Proc. Amer. Math. Soc. (to appear).

[Bá] I. Bárány, A short proof of Kneser's conjecture, J. Combin. Theory Ser. A 25 (1978), 325-326.

[BKL] A. Björner, B. Korte, and L. Lovász, Homotopy properties of greedoids, Adv. in Math. (to appear).

[B] B. Bollobás, On complete subgraphs of different orders, Math. Proc. Cambridge Philos. Soc. 79 (1976), 19-24.

[Bo] K. Borsuk, On the embedding of systems of compacts in simplicial complexes, Fund. Math. 35 (1948), 217-234.

[Bou] D. G. Bourgin, Modern algebraic topology, Macmillan, New York; Collier-Macmillan, London, 1963.

[BSS] I. Bárány, S. B. Shlosman, and A. Szücs, On a topological generalization of a theorem of Tverberg, J. London Math. Soc. (2) 23 (1981), 158-164.

[CL] E. J. Cockayne and P. J. Lorimer, The Ramsey numbers for stripes, J. Austral. Math. Soc. (Ser. A) 19 (1975), 252-256.

[E] P. Erdös, Problems and results in combinatorial analysis, Colloq. Internat. Theor. Combin. Rome 1973, Acad. Naz. Lincei, Rome, 1976, pp. 3-17.

[ER] P. Erdös and R. Rado, Intersection theorems for systems of sets, J. London Math. Soc. 35 (1960), 85-90. 
[EKR] P. Erdös, C. Ko, and R. Rado, Intersection theorems for systems of finite sets, Quart. J. Math. Oxford 12 (1961), 313-320.

[ES] P. Erdös and M. Simonovits, Supersaturated graphs and hypergraphs, Combinatorics 3 (1983), 181-192.

[F1] P. Frankl, On intersecting families of finite sets, J. Combin. Theory Ser. A 24 (1978), 146-161.

[F2] _ On the chromatic number of general Kneser graphs, J. Graph Theory 9 (1985), 217-220.

[FF] P. Frankl and Z. Füredi, Extremal problems concerning Kneser graphs, J. Combin. Theory Ser. B 40 (1986), 270-284.

[FR] P. Frankl and V. Rödl, Hypergraphs do not jump, Combinatorica 4 (1984), 149-159.

[Gy] A. Gyárfás, On the Ramsey number of disjoint hyperedges, J. Graph Theory (to appear).

[GW] C. H. Goldberg and D. B. West, Bisection of circle colorings, SIAM J. Algebraic Discrete Methods 6 (1985), 93-106.

[HR] A. Hajnal and B. L. Rothschild, A generalization of the Erdös-Ko-Rado theorem on finite set systems, J. Combin. Theory Ser. A 15 (1973), 359-362.

[KNS] G. Katona, T. Nemetz, and M. Simonovits, On a graph problem of Turán, Mat. Lapok 15 (1964), 228-238.

[Kn] M. Kneser, Aufgabe 300, Jber. Deutsch. Math.-Verein. 58 (1955).

[L1] L. Lovász, Kneser's conjecture, chromatic number and homotopy, J. Combin. Theory Ser. A 25 (1978), 319-324.

[L2] - Self dual polytopes and the chromatic number of distance graphs on the sphere, Acta Sci. Math. (Szeged) 45 (1983), 317-323.

[T1] P. Turán, On an extremal problem in graph theory, Mat. Fiz. Lapok 48 (1941), 436-452.

[T2] _, On the theory of graphs, Colloq. Math. 3 (1954), 19-30.

Department of Mathematics, Tel Aviv University, Tel Aviv, Israel

Bell Communications Research, 435 South Street, Morristown, New Jersey 07960

Département de Mathématiques, Université de Paris VII, Paris, France

AT\&T Bell laboratories, 600 Mountain Avenue, Murray Hill, New Jersey 07974

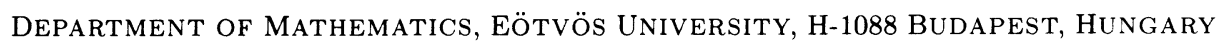

